\title{
Keep breathing
}

\section{stimulation of mid-cervical elNs in mice ... resulted in a substantial recovery of respiration- related diaphragm activity}

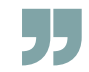

Most deaths after spinal cord injury (SCI) occur owing to respiratory complications arising from damage to neuronal networks that innervate the diaphragm to regulate breathing. The Fehlings team (Satkunendrarajah et al.) now show that recruitment of a population of excitatory interneurons (eINs) in the cervical spinal cord of mice sustains respiratory function after non-traumatic SCI and can be activated to restore breathing after traumatic SCI.

Traumatic injury to the cervical spinal cord disrupts the descending pathways that drive phrenic motor neuron (PMN) activity, leading to respiratory dysfunction. Non-traumatic SCI, on the other hand, does not typically cause respiratory problems, despite evidence of considerable PMN damage. The authors therefore used a mouse model of a form of non-traumatic SCI (ntSCI) called cervical myelopathy, in which the spinal cord is compressed at the mid-cervical (C4-C6) level, to investigate the mechanisms by which respiratory function may be sustained.

In line with previous findings, the authors reported a $64 \%$ loss of PMNs in ntSCI mice by 8 weeks after injury. Nevertheless, these mice

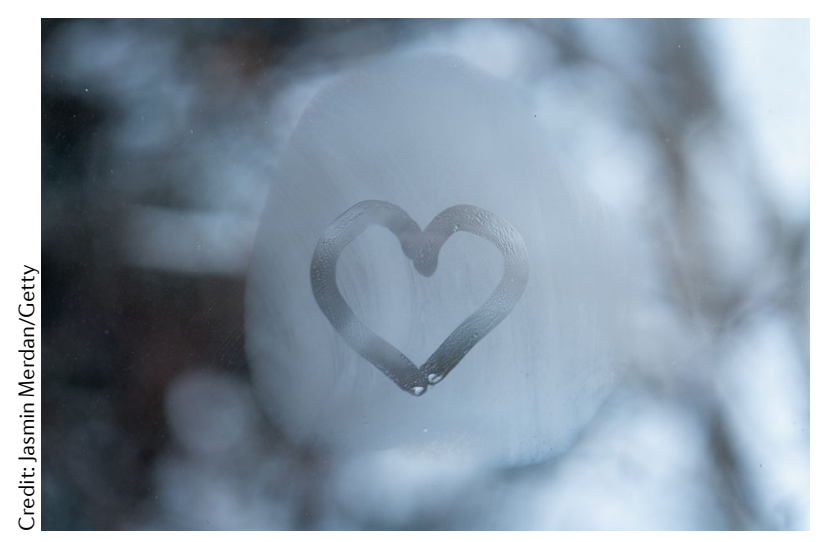

displayed only minor respiratory changes when compared with control (sham-operated) mice, indicating that plasticity in the spinal neural networks that regulate breathing could sustain respiratory function.

The plastic changes induced in ntSCI mice were also sufficient to maintain respiratory function after a subsequent traumatic SCI: the authors showed that ntSCI mice subjected to a spinal cord hemisection at the $\mathrm{C} 2$ level did not suffer the immediate and total loss of inspiration-related diaphragm activity observed in controls.

These findings suggest that changes to local microcircuits take place after ntSCI and act to compensate for the loss of normal PMN function. Using trans-synaptic tracing and immunohistochemistry, the authors observed an increase in the number of connections made by excitatory interneurons (eINs) on surviving PMNs at the mid-cervical level in ntSCI mice, indicating that these neurons may be recruited to sustain respiratory activity.

To investigate the contribution of mid-cervical eINs to breathing after SCI, the authors used a viral vector to selectively express a chimeric receptor-chloride channel in this neuronal population. In ntSCI mice, the activation of this channel, causing transient inhibition of eINs, markedly reduced inspiration amplitude. In addition, it prevented the maintenance of respiratory activity after $\mathrm{C} 2$ hemisection in these mice. Furthermore, in control mice subjected to $\mathrm{C} 2$ hemisection, acute inhibition of eIN activity interrupted the spontaneous recovery of respiratory function that is normally observed 2-4 weeks after injury, confirming the importance of cervical eINs for breathing recovery after SCI.

These results suggest that strategies to boost the recruitment or activity of cervical eINs in the period immediately following injury (when most respiration-related deaths occur) might be exploited to improve outcome after traumatic SCI. Indeed, the authors demonstrated that pharmacogenetic stimulation of mid-cervical eINs in mice immediately following a $\mathrm{C} 2$ hemisection resulted in a substantial recovery of respiration-related diaphragm activity in these animals.

The findings also provide insight into the role of cervical eINs in the absence of injury. The transient inhibition of the activity of these neurons in uninjured control mice had little effect on overall respiratory activity, indicating that these neurons are not essential for normal breathing. Chemogenetically activating these neurons was sufficient to increase inspiratory amplitude but did not alter the inspiration frequency, suggesting that cervical eINs can modulate PMN activity, but do not provide input to the higher brain centres that regulate the pattern of respiratory activity.

This study has identified a population of spinal interneurons that have a crucial role in the recovery of respiratory function after injury and that may present an amenable target for both experimental manipulation and therapeutic efforts to reduce patient mortality after traumatic SCI.

Katherine Whalley

ORIGINAL ARTICLE Satkunendrarajah, K. et al. Cervical excitatory neurons sustain breathing after spinal cord injury. Nature https://doi.org/ 10.1038/s41586-018-0595-z (2018) 